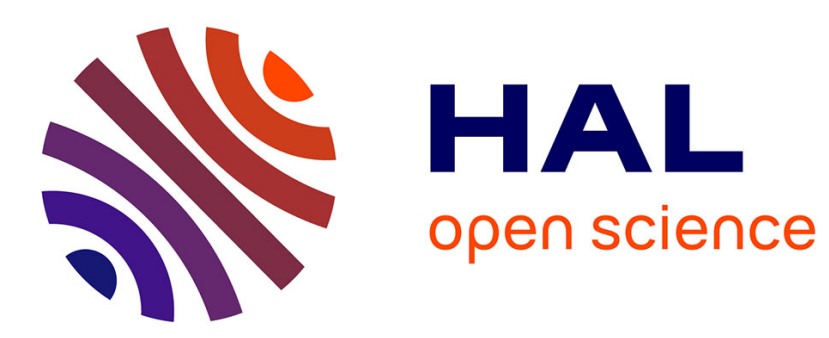

\title{
La légitimité culturelle en questions
}

Emmanuel Ethis, Emmanuel Pedler

\section{To cite this version:}

Emmanuel Ethis, Emmanuel Pedler. La légitimité culturelle en questions. Le travail sociologique de Pierre Bourdieu - Dettes et critiques, VII, La Découverte, pp.178-204, 1999, Textes à l'appui. halshs-00007310

\section{HAL Id: halshs-00007310 https://shs.hal.science/halshs-00007310}

Submitted on 17 Dec 2005

HAL is a multi-disciplinary open access archive for the deposit and dissemination of scientific research documents, whether they are published or not. The documents may come from teaching and research institutions in France or abroad, or from public or private research centers.
L'archive ouverte pluridisciplinaire HAL, est destinée au dépôt et à la diffusion de documents scientifiques de niveau recherche, publiés ou non, émanant des établissements d'enseignement et de recherche français ou étrangers, des laboratoires publics ou privés. 


\title{
La légitimité culturelle en questions
}

\author{
par Emmanuel Ethis \\ et Emmanuel Pedler
}

Sur le marché des idées, les théories explicatives globales rendant compte de l'histoire humaine, d'événements, de situations, de comportements ou d'attitudes en leur part sociale et culturelle affleurent régulièrement, s'imposent durant une période plus ou moins longue, puis subissent les assauts d'une critique argumentée qui en relativise la portée. Pour être convainquantes, elles supposent chez leurs auteurs l'alliance de qualités rares. Il faut des qualités plus rares encore lorsque les gains d'intelligibilité ainsi obtenus s'appuient sur une description respectueuse de la complexité et de la diversité du monde social. Cet état de grâce de la pensée advient parfois, mais est suffisamment peu fréquent pour susciter la fascination qu'appelle toute haute virtuosité ; il s'inscrit dans un genre que nous dirons " héroïque" et qui s'éloigne du droit commun régissant l'activité de recherche en sciences sociales pour lequel la complexité énonciative épouse l'univers bariolé des choses.

Nous ne débattrons pas ici - car nous n'en avons pas les moyens - du fait de savoir s'il peut exister un optimum pour lequel une intelligibilité maximale et une descriptivité fine peuvent cohabiter. Constatons simplement que pour le domaine qui nous occupe, la sociologie de la culture, les théories globalisantes ont été le plus souvent prises en défaut et se sont heurtées à diverses objections. Il n'y donc rien d'étonnant à ce que la théorie de la légitimité suscite aujourd'hui, y compris de la part de ceux qui s'en sont nourri, le sort commun et soit soumise à la critique.

Ce faisant les cheminements de l'objection ont emprunté des voix diverses : celle de la théorie pure - nous pensons ici à la théorie de l'action, aux travaux engagés dans le sillage des Economies de la grandeur par exemple — ; celle de l'empirie, qui, confrontée aux objets culturels les plus divers, éprouve la plasticité et l'efficacité explicative d'un cadre théorique apparemment très adaptable. C'est dans cette dernière voie que nous engagerons notre discussion. Ce faisant et sans qu'il soit besoin de présenter ici une synthèse reprenant le fil, bien connu, qui conduit des Héritiers, de la Reproduction, de l'Amour de l'art aux Règles de l'art, en passant par la Distinction, nous souhaitons mettre l'accent sur deux ordres de faits : (1) le lien problématique qui existe entre les variables qui cherchent à rendre compte des comportements culturels - comme la formation scolaire, l'appartenance à un " milieu" — et ces comportements, (2) la nature des indicateurs à partir desquels la théorie de la légitimité appréhende ces derniers déclarer une pratique culturelle, formuler un jugement ou "le révéler" par ses actes et ses attitudes. Nous renverrons ici à des travaux que nous résumons brièvement 
La légitimité culturelle en questions - 2

dans le but de pointer la nature des objections qui nous semblent faire sens, un article bref, comme celui-ci ne permettant pas de rentrer plus avant dans le détails des arguments avancés.

Ajoutons pour finir que nous ne chercherons pas ici à inscrire les paradoxes et les limites d'une théorie dans un cadre théorique englobant. Aux constructions de tailles plus modestes vont nos préférences.

\section{La légitimité culturelle en questions (1/2)}

\section{La liaison problématique entre formations scolaires et pratiques culturelles: de l'appartenance géographique à l' "l'effet paradoxal du niveau de diplôme"}

On ne peut dissocier la question de la démocratisation culturelle du culte qui valorise et qualifie les productions artistiques en leur donnant un statut patrimonial.

Il faut préciser d'entrée de jeu ce que nous entendons par "culte" afin d'explorer ensuite les fondements argumentatifs et empiriques des thèses qui, à partir de l'analyseur qu'est la notion de démocratisation culturelle, tentent de rendre compte des inégalités sociales face aux objets culturels. La vision enchantée de l'art appelle des analyses sur lesquelles il n'est pas possible de s'étendre trop longuement: on retiendra seulement une définition, fatalement provisoire, de ce supplément d'âme, de cette plus-value décisoire qui est constitutive d'un rapport enchanté aux œuvres de l'esprit. On dira que cette vision est attestée lorsqu'une production de l'esprit cesse d'être perçue comme constituée d'éléments hétérogènes et lorsqu'on la reçoit comme formant un Tout indécomposable. A l'inverse, lorsque cet enchantement se dissout, un rapport que l'on dira critique — c'est-à-dire relativiste - confère aux productions humaines un aspect provisoire et fatalement inachevé. On apprend ainsi, dans les formations à la recherche, à désassembler les arguments et les "preuves" empiriques pour considérer, par exemple, que La Distinction ou tout autre ouvrage sociologique, constitue une thèse dont on peut discuter les attendus, les arguments et les bases empiriques, pour faire apparaittre leur relativité, pour reconstituer les trajectoires historiques des idées, arguments et modes de preuve à l'œuvre dans ce texte. Remarquons que, pour les objets artistiques, il n'en va pas exactement de même, puisqu'un 


\section{La légitimité culturelle en questions - 3}

des espoirs de l'artiste et de ceux qui le secondent consiste justement à donner l'illusion que les éléments disparates dont il se sert ont fusionné dans un ensemble organique ${ }^{1}$. Pour ces raisons, les objets artistiques et les œuvres de l'esprit constituent des catégories pouvant susciter des rapports très variables : bien rares ou banalisés, luxueux ou cultuels selon les cas, ils constituent des cas limites pour les théories politiques de la culture.

On voit ici la restriction que doit subir l'analyse de ces objets pour justifier les théories selon lesquelles les œuvres de l'esprit et de l'art, les savoirs, les artisanats d'art apparaîtraient comme autant de biens rares et luxueux qu'il importerait de mettre à la disposition de tous. Pour une telle vision, les efforts consentis pour acquérir, restaurer et susciter ce qui se nomme aujourd'hui "la création" — surtout lorsque ces efforts sont réalisés à partir de fonds publics - devraient démocratiquement recevoir pour contrepartie une pénétration sociale équitable, c'est-à-dire repartie en fonction du poids relatif des ensembles sociaux qui composent la nation.

Essayons, fictivement et temporairement, de nous placer dans cet espace argumentatif en levant les restrictions que nous venons d'énoncer. Supposons donc que les objets artistiques sont des biens rares et luxueux, produits, créés et reproduits par la collectivité et que cette dernière demande des comptes des dépenses consenties.

Le premier constat serait dès lors le suivant : la redistribution s'effectue mal, très mal. La façon la plus efficace de la mesurer, à un niveau national ou européen ${ }^{2}$ est d'évaluer les effets tendanciels des formations scolaires sur les pratiques culturelles. Cette variable est apparue en effet, depuis longtemps, comme étant particulièrement sensible et réactive en sociologie de la culture. Elle est plus efficace que d'autres indicateurs d'identité sociale, de confessions, de générations, de sexe, de milieu social puisque ces dernières variables ne possèdent jamais en comparaison qu'une puissance marginale. Pourtant l'efficacité tendancielle des niveaux de formation scolaire est d'un maniement difficile, car, dès qu'on en neutralise la dimension comparative - les plus diplômés ont tendance, plus que les autres, à s'intéresser aux offres culturelles savantes — pour décrire les milieux sociaux qui

\footnotetext{
1. A ce stade de l'analyse, on peut remarquer que les thèses de la légitimation par une institution, une instance ou un personnage charismatique ne sont qu'une exploration spécifiquement sociologique d'un phénomène plus général qui relève de l'anthropologie culturelle.
} 


\section{La légitimité culturelle en questions - 4}

entretiennent un rapport régulier avec ces offres, l'effet se dissipe. On peut ainsi montrer que les cultures savantes ne constituent pas la culture dominante des groupes dits "supérieurs", , même si ces derniers, proportionnellement, sont plus nombreux à entretenir un rapport — souvent très distant — avec ces offres.

Si nous continuons à nous maintenir dans la fiction d'un modèle redistributif, nous pouvons encore faire le constat qu'il existe des offres et des dispositifs qui, plus que d'autres, favorisent ou défavorisent une "démocratisation" culturelle. Or ces offres et dispositifs révèlent l'existence de filtres très efficaces qui ne recoupent pas les variables classiques le plus souvent testées et comparées à la variable miraculeuse du niveau de diplôme. Le huis clos de l'arbitrage comparatif entre variables explicatives s'effectue donc sous une cloche de verre qui en limite la valeur de la comparaison. C'est là un point sur lequel nous nous arrêterons dans un second temps.

En dernier lieu, si la plupart des enquêtes réalisées en Europe, mais également aux Etats-Unis ${ }^{4}$ font apparaitre une corrélation selon laquelle plus le niveau de formation scolaire est élevé, plus les pratiques culturelles s’intensifient, il suffit de changer la nature des indicateurs mesurant le rapport aux offres et aux "œuvres" pour constater divers dérèglements paradoxaux. C'est ce qu'à permis de révéler une enquête consacrée au face à face entre un public de musée et les toiles exposées ${ }^{5}$. Ce sont ainsi les sujets dotés d'une formation universitaire courte qui consacrent le plus de temps au musée et sont porteurs, comparativement aux autres groupes plus ou moins formés au plan scolaire, de la plus grande "appétence” et compétence culturelles.

\footnotetext{
2. JM Guy, Documentation française, 1991.

${ }^{3}$. Emmanuel Pedler, Sociologie de l'opéra, Parenthèses, octobre 1999.

${ }^{4}$. E. Pedler, Sociologie de l'opéra, Parenthèses, oct. 1999.

5. Jean-Claude Passeron et Emmanuel Pedler, Le temps donné aux tableaux, I.Me.Re.C., 1991. Voir également "Le temps donné au regard ", in Théories de la réception, Chicoutimi : Protée, juin 1999, p.x à p.x.
} 
La légitimité culturelle en questions - 5

\section{"Classes sociales" et cultures savantes au XXe siècle}

La lecture de nos statistiques nationales — sur une profondeur d'une vingtaine d'années au moins — de résultats comparables collectés dans différents pays européens ou aux Etats-Unis, donne régulièrement une formule gagnante : les "classes dominantes" contrôles et bénéficient le mieux des patrimoines savants pieusement conservés dans les théâtres lyriques, les musées, les salles de concerts ou les bibliothèques publiques, etc. Ainsi ces lieux seraient tenus et fréquentés par la mince couche sociale la plus favorisée et bénéficieraient pour continuer à exister du soutien massif des fonds publics. Ce constat se prête, à tout le moins, à deux objections. La stratification encore nette de notre société contemporaine permet-elle encore de parler de classes dominantes? Les pratiques savantes sont-elles dominantes au sein des groupes supérieurs? La première objection n'appelle pas longs commentaires sinon pour constater l'important et constant élargissement des groupes "moyens" et l'éclatement partiel des appartenances culturelles ${ }^{6}$. On peut donc douter sérieusement que la tripartition sociale de nos sociétés contemporaines en classes populaire, moyenne et supérieure soit encore pertinente. Mais ce n'est pas sur ce plan qu'il est le plus urgent d'engager un nouvel examen de nos statistiques nationales. Les tendances qui dessinent des corrélations nettes entre pratiques de sortie - à l'opéra, au concert, au musée, etc. - et les fractions supérieures de la population française sont à analyser à la lumière des chiffres absolus de ces pratiques. Or ces derniers sont dérisoires. Seule une fraction minoritaire de ces groupes supérieurs est concernée par les pratiques culturelles.

Un fait supplémentaire accentue encore cet état : les fractions sociales supérieures qui s'adonnent aux pratiques culturelles ne constituent pas une sous-population représentative de l'ensemble auquel elles appartiennent. Pour l'apercevoir, il faut accepter d'embrasser d'un seul regard la totalité des populations qui constituent - par position relative - les groupes supérieurs de la société française contemporaine. Si l'on prend les catégories de l'INSEE, il apparaît que les "professions intellectuelles supérieures" possèdent en leur sein des segments dont les représentants n'émargent que faiblement aux pratiques cultivées. Ce

\footnotetext{
6. Voir notamment Revue Française de Sociologie, mars 1999.
} 


\section{La légitimité culturelle en questions - 6}

sont ainsi les professions du secteur public qui témoignent le plus d'allant et d'appétence face à l'offre théâtrale du festival d'Avignon ${ }^{7}$. Dans le cadre du pôle régional qui constitue l'écrin démographique naturel des pratiques culturelles de sortie — car l'offre est polarisée par quelques villes phares - les habitués des musées, les notables proches des théâtres lyriques et des salles de concerts ou les abonnés de théâtre ne constituent pas un échantillonnage non biaisé des groupes dits "supérieurs". Sur ce plan, il faudra en sociologie de la culture, renouveler les cadres d'analyse et tester les propositions les plus diverses. Ainsi lorsque Jean-Claude Milner dans Le salaire de l'idéaß oppose bourgeoisies du surtemps et du sursalaire ${ }^{9}$, il nous fait entrevoir la frontière qui sépare les fractions dominantes riches de leurs temps de leurs homologues qui disposent de revenus importants. Comme ces dernières - qui comprennent une partie des professions libérales - ne manifestant pas plus d'allant en matière culturelle que le reste de la population française $^{10}$, il pourrait être judicieux de renouveler les découpes classiques opposant les niveaux de formation scolaire ou les appartenances à des milieux, pour tester de nouvelles catégories.

Reste que pour ces ensembles sociaux minoritaires, les consommations de l'art sont le plus souvent indolentes et inchoatives, car elles portent sur des objets qui ne possèdent aucune unité culturelle. Les répertoires des principaux théâtres lyriques, des salles de concert ou de grandes expositions sont artificiels ; collection d'objets hétéroclites coupés de leur histoire, les "grandes CEuvres" du répertoire — dont les dictionnaires de la musique fortifient d'édition en édition l'inébranlable stabilité - ne constituent pas un objet homogène de pratique. Lorsque l'on réintègre une œuvre issue de ce sérail dans la parentèle

7. Emmanuel Ethis, Jean-Louis Fabiani, Emmanuel Pedler, Les publics du festival d'Avignon I, Spectateurs, identités et trajectoire, Documentation Française, Juin 1999.

${ }^{8}$. Jean-Claude Milner, Le salaire de l'idéal, La théorie des classes et de la culture au XXe siècle, Seuilessais, 1997.

9. Il faut s'empresser d'ajouter que cela n'est pas le cas de l'ensemble des propositions que renferne ce livre. On aurait en effet bien du mal ^ concilier la théorie de la culture de Jean-Claude Milner avec les faits que relève l'enquête.

${ }^{10} \mathrm{Il}$ faut ajouter que ni les radiographies précises de l'INSEE, ni les monographies très ciblées des auteurs les plus écoutés sur le sujet10, n'offrent de prises réelles pour discuter de ce problème puisque la fracture qui traverse l'univers bourgeois - acceptons l'expression, le temps de notre discussion — ne peut ni s'apercevoir à partir des découpages professionnels, ni se circonscrire par une approche monographique qui cible une sous-population singulière. 


\section{La légitimité culturelle en questions - 7}

dont elle est issue, pour un auditeur aguerri ou pour un praticien, elle cotoie tout un demimonde bariolé. C'est ainsi que, pour le concertiste, une partita de Bach pour violon seul cohabite avec les pièces brillantes de Sarasate, Vieuxtemps ou Wieniawski ou avec la virtuosité subtile d'un Ysaye. L'ethnographie des milieux musicaux contemporains fait apparaître un étonnant mélange des genres et des goûts qui ne peut se comparer qu'à la brocante esthétique des amateurs ordinaires, lorsqu'ils font autre chose que de saluer respectueusement une offre qu'ils ne fréquentent pas intimement. Si l'on adopte un point de vue génétique pour s'interroger sur les CEuvres d'accompagnement qui ont escorté une pièce aujourd'hui classée et sortie de son contexte, le même constat saute aux yeux. Hors de quelques cas d'espèce ${ }^{11}$, les pièces rangées aujourd'hui dans nos panthéons sont devenues des vaisseaux fantômes. Elles s'offrent ainsi, non sans ambiguïtés, à un vaste public, forcément démuni et dérouté.

Il faut enfin prendre en compte une donnée que l'histoire culturelle met rarement en évidence. La "bourgeoisie intellectuelle" qui cultive avec le plus de conséquence - au moins tendantiellement - les valeurs culturelles savantes ne saurait être tenue pour l'héritière des aristocraties et grandes bourgeoisies du siècle passé. Les grandes cathédrales mondaines du début du siècle — la salle Garnier en est un exemple — enregistrent dans les années 1920 la montée en puissance des bourgeois salariés et, concurremment, la chute inexorable des groupes jusque-là dominants. Dans le monde de l'opéra, le foyer, lieu de rassemblement du "tout Paris" voit ainsi peu à peu ses frontières s'affaisser sous la pression de figures étrangères au $M_{0}{ }^{12}$. A ce même moment, les répertoires changent en s'éloignant des valeurs académiques prisées jusque-là. Les artistes et leurs cercles acquièrent à ce moment une autonomie accrue, s'appuient sur des forces plus diversifiées et imposent des critères de goût plus complexes. L'objet d'art n'est plus seulement un objet de luxe. Ainsi la hiérarchie sociale et la hiérarchie des œuvres cessent de s'articuler simplement.

C'est sans doute là que réside la justification de la marginalisation, de l'éclatement et du fractionnement des pratiques cultivées. C'est la raison pour laquelle les appartenances

\footnotetext{
${ }^{11}$. Il est bien sûr possible de chercher des contre exemples; dans le domaine lyrique, le Pélléas de DEBUSSY est ainsi une sorte de météorite sans ascendance, mais dont la carrière sociale — c'est-à-dire la domestication hors du cercle étroit de quelques fractions du clergé culturel - a été singulièrement courte, pour ne pas dire inexistante.
} 
La légitimité culturelle en questions -8

géographiques — qui sédimentent des histoires culturelles singulières, tant au niveau de l'offre qu'au plan de la demande - peuvent apparaitre, en certains cas, d'une plus grande pertinence pour expliquer la forme que prend la fréquentation des œuvres savantes.

\section{Eloignement et proximité, les filtrages sociodémographiques de la fréquentation : le cas du Festival d'Avignon}

Depuis les années quatre vingt dix, les enquêtes nationales tendent à ne plus être le modèle unique d'exploration afin d'évaluer les pratiques culturelles en France. Des enquêtes réalisées ou soutenues par le Département d'Etudes et de la Prospective du Ministère de la Culture, ont ainsi peu à peu abandonné le cadre national qui servait de référence. On peut citer à titre d'exemple, l'étude portant sur Les publics de la Comédie Française $^{13}$, réalisée sous la direction de Jean-Michel Guy et publiée en 1997. Nous nous appuierons quant à nous sur l'enquête que nous avons engagée, grâce à l'aide du D.E.P., afin de décrire les publics du festival d'Avignon et les rapports qu'ils entretiennent avec l'offre.

En 1996, 0,7\% de l'échantillon prélevé en Avignon, lors du cinquantenaire du Festival, appartenait au monde ouvrier. Le constat est banal et il est presque inutile de relever que ce chiffre n'a que peu de rapport avec les pourcentages de cette catégorie professionnelle dans la population active, nationale ou avignonnaise. Le festival d'Avignon, comme la plupart des festivals, n'est pas un festival populaire. Pourtant l'analyse sociodémographique du festival montre que son public s'est diversifié.

C'est en faisant varier les origines géographiques des festivaliers qu'apparaissent des signes clairs d'ouverture. Tout se passe comme si le festival effectuait une filtration sociale d'autant plus marquée que les publics sont éloignés du cercle local (Vaucluse et Bouches du Rhône qui apportent environ $20 \%$ de la population festivalière). Ce qui revient à remarquer que le festival sur-sélectionne (par le haut) d'autant plus ses spectateurs que ceux-ci

\footnotetext{
${ }^{12}$. Patureau, La salle Garnier, Mardaga

13 . Paris : La Documentation Française, 1997.
} 


\section{La légitimité culturelle en questions -9}

viennent de $\operatorname{loin}^{14}$. Il faut ajouter que cette situation ne peut se réduire à une mécanique kilométrique, la zone la plus "éloignée” du cercle local étant ici la région parisienne. Jusquelà, on ne peut rien constater de franchement inattendu: comme différents dispositifs, scolaires, notamment, une offre prestigieuse ne réussit à mobiliser au sein des prétendants les plus éloignés que la part la plus impliquée, la plus mobilisée ${ }^{15}$.

Les attentes, les rythmes festivaliers, les œÒuvres fréquentées changent ainsi progressivement lorsque l'on s'éloigne du cercle local. Tout laisse à penser que le festival n'est plus le même lorsqu'on est placé aux deux extrémités de ce continuum géographique. Manifestation de prestige goûtée lors d'un séjour d'une durée moyenne (entre 3 et 8 jours) et onéreux, pour les fractions parisiennes à hauts revenus et hauts diplômes, le festival devient pour les fractions locales un lieu de pèlerinage (présence à de nombreuses éditions antérieures de la manifestation). Mais il est paradoxal, parce qu'inattendu si l'on se place du point de vue de la théorie de la légitimité, que le public local soit celui qui explore le plus studieusement l'offre présentée : les spectacles "in" vus sont beaucoup plus nombreux pour les fractions possédant une formation universitaire brève, elles manifestent des intérêts plus diversifiés, une plus grande familiarité avec le théâtre et une réelle curiosité en direction de l'offre moins instituée du "off".

Il faut ajouter que les filtres qui conduisent ainsi à sculpter les publics en fonction de leur proximité ou éloignement au centre parisien sont divers et complexes. Les fortifications qui ne laissent passer que peu de monde à mesure que l'on s'éloigne du cercle local sont constituées pour une part — et le fait est banal — par des freins économiques et culturels. Il faut en effet aux fractions sociales des publics potentiels de la région parisienne une forte mobilisation pour franchir les différentes barrières — éloignement, problèmes d'intendance sur les lieux du festival, etc. — mais l'effet progressif des "filtres" — qui jouent plus fortement pour la Région parisienne que pour les autres départements hors PACA, plus pour ces derniers que pour la Région PACA, etc. — ne se laisse pas expliquer si simplement. Il faut en effet se souvenir que la carte des publics potentiels ne se confond

\footnotetext{
14. Encore est-ce là une façon de s'exprimer, puisque aucun dispositif volontaire n'est à l'origine de ce phénomène.

15. On peut penser, notamment, à l'enquête Rapport pédagogique et communication, qui révélait une économie assez comparable à celle que nous observons ici.
} 


\section{La légitimité culturelle en questions - 10}

pas avec celle des densités de population. Ce ne sont pas les départements les plus peuplés qui envoient le plus de spectateurs et en conséquence la géographie culturelle ne peut tenir compte seulement des seules données démographiques, physiques - comme l'éloignement - ou économiques. Le rapport aux Cuvres, la fidélité au festival, la permanence de l'intérêt pour les pièces présentées sur une période plus ou moins longue sont autant de modalités qui varient puissamment selon qu'on est avignonnais ou parisien ; la sociodémographie n'épuise donc pas la question.

Pour l'essentiel, on peut retenir les trois constats suivants : (1) les publics du cercle local et régional cumulent des caractéristiques propres très notables : ouverture des origines socioprofessionnelles et des conditions économiques, singularité des comportements festivaliers et du rapport aux pièces du "in"; (2) ces fractions ne sont pas marginales (elles constituent environ $40 \%$ de l'échantillon) ou composées de publics estudiantins (y sont surreprésentés les spectateurs ayant entre 35 et 50 ans, ceux exerçant des professions intermédiaires et des employés; (3) les attitudes culturelles du cercle local - et partant leurs attentes, leurs intérêts et leurs jugements - ne peuvent être prédites et fondées exclusivement sur les formations scolaires ou universitaires des personnes qui la composent.

Bref, on le voit, en choisissant de déconstruire l'artefact que constitue la grille des appétences tendancielles pour la culture, on se donne les moyens de révéler des situations singulières susceptibles d'invalider les explications globalisantes et par trop mécaniques des comportements culturels.

\section{L'effet paradoxal du diplôme}

Nous l'avons dit, depuis L'Amour de l'art ${ }^{16}$, la sociologie de la fréquentation du Musée s'est habituée à retenir le niveau d'instruction comme variable principale, parfois unique, de toute variation d'un comportement culturel, en tout cas comme une variable dont l'action serait “ linéaire”. Or, si l'on s’intéresse à la mesure la plus globale du coût consenti par les visiteurs pour regarder de la peinture — pour l'enquête à laquelle nous nous référons ici, Le

\footnotetext{
${ }^{16}$ P. BOURDIEU et A. DARBEL, Paris, Minuit, 1970.
} 


\section{La légitimité culturelle en questions - 11}

temps donné aux tableaux, le temps qu'ils consacrent au musée, entre l'entrée et la sortie - les variations du temps donné au musée en fonction des différents niveaux de diplôme révèlent, et cela est classique, une corrélation statistique. Mais elle est en ce cas d'une forme bien particulière, que les auteurs ont qualifier d'effet paradoxal du niveau de diplôme sur le comportement de visite d'un Musée, dès lors que l'on s'attache à distinguer au-delà du Baccalauréat deux longueurs d'études (études supérieures courtes et longues).

“Comme on peut le voir dans le tableau qui suit, notent les auteurs, les sujets les moins diplômés se retrouvent dans la zone moyenne de l'indice mesurant le temps donné au musée, tandis que les diplômés moyens de l'enseignement supérieur sont représentés plus que proportionnellement dans la zone haute de l'indice du temps donné au musée.

Le temps donné au musée (indice) selon le diplôme

\begin{tabular}{|l|l|l|l|l|}
\hline $\begin{array}{l}\text { Indice } \\
\text { Diplôme }\end{array}$ & Inf. à 30’ & Entre 30’ et 47’ & $\begin{array}{l}\text { Au-dessus } \\
\text { De 48’ }\end{array}$ & Total \\
\hline
\end{tabular}

\begin{tabular}{|l|l|l|l|l|}
\hline Inférieur au Bac. & $23 \%$ & $\mathbf{5 1 \%}$ & $26 \%$ & $100 \%$ \\
\hline Bac. à Bac. +3 & $28 \%$ & $39 \%$ & $\mathbf{3 3 \%}$ & $100 \%$ \\
\hline Bac+4 et + & $\mathbf{4 0 \%}$ & $36 \%$ & $24 \%$ & $100 \%$ \\
\hline
\end{tabular}

\begin{tabular}{|l|l|l|l|l|}
\hline Ensemble & $31 \%$ & $41 \%$ & $28 \%$ & $100 \%$ \\
\hline
\end{tabular}

Ce sont donc, paradoxalement, les sujets les plus hautement diplômés qui sont proportionnellement les plus représentés au niveau faible de l'indice (ce que souligne ici en gras de la plus forte tendance par colonne). Autrement dit, en allant des temps les plus brefs passés dans le musée aux temps les plus longs, on rencontre d'abord (parmi les plus représentés) les sujets les plus diplômés, puis les moins diplômés avant de rencontrer les sujets moyennement diplômés qui sont ceux qui passent le plus de temps dans le musée. ${ }^{17,}$ On retrouve le même ordre inattendu " en caractérisant les visiteurs par le temps moyen donné au musée par leur catégorie de diplômes, 
Le temps moyen passé dans le musée selon le diplôme

\begin{tabular}{|l|l|l|l|l|}
\hline Diplôme & Inf. au Bac. & Bac. à Bac. +3 & Bac. +4 et + & Total \\
\hline Temps moyen & $41^{\prime} 16 ”$ & $43^{\prime} 28^{\prime \prime}$ & $38^{\prime} 08^{\prime \prime}$ & $40^{\prime} 54^{\prime \prime}$ \\
\hline
\end{tabular}

L'ordre croissant des temps moyens ordonne les diplômes dans l'ordre 3,1,2. Cette structure récurrente est bien celle qui caractérise en fonction du diplôme le temps global que les visiteurs donnent au musée. On voit directement sous une forme graphique cette relation paradoxale sur un histogramme : il faut désordonner l'ordre scolaire pour que l'histogramme présente une pente régulière correspondant à l'augmentation du temps donné au musée. ${ }^{18}$,

Les habitudes scolaires engendrent, un temps au moins, une docilité envers les hiérarchies culturelles. Mais les disciplines mentales enseignées par l'Ecole procèdent à l'inverse d'un mouvement qui peut être au fondement d'attitudes critiques et non conformistes. L'efficacité du marquage scolaire ne se résume pas à une action mécanique et régulière. La relation entre la hiérarchie des diplômes ou des longueurs d'étude et ses effets se trouve, selon les effets qu'on analyse, prendre tantôt la forme "classique", tantôt la forme "paradoxale", faisant ainsi apparaître la complexité d'une situation qui ne se laisse pas facilement synthétiser sous la forme mécanique proposée dans la "théorie de la légitimité", telle qu'elle est exposée dans La Distinction, par exemple.

Le bilan relativiste que nous venons de dresser nous permet de conclure en insistant sur l'urgence qu'il y a à refuser toute simplification dans la description des pratiques culturelles. Le mérite de la première grande enquête qui fit passer durablement le critère scolaire au premier plan tenait à l'existence, pour cette recherche, d'un réel effort descriptif $^{19}$. Le rapport des étudiants à la langue d'idée enseignée par l'école, mesuré grâce à des indicateurs couvrant tous les registres de cette langue - des malaproprismes qui

${ }^{17}$. Le temps donné aux tableaux, Op.Cit., p.X.

${ }^{18}$. Op. Cit., p.X.

${ }^{19}$. Bourdieu (P.), PASSERon (J.-C.) et DE SAINT-MARTin (M.), Rapport pédagogique et communication, Paris/La Haye : Mouton, 1965. 
objectivent un rapport verbeux ${ }^{20}$ à la langue, aux exercices de polysémie ou de définition, en passant par les lexiques humanistes et techniques — était de nature à révéler l'emprise d'un des instruments cardinaux de l'inculcation scolaire, la langue d'enseignement. D'extrapolations en extrapolations, le schème d'analyse qui était au fondement de la "théorie de la légitimité" a beaucoup gagné en intelligibilité ; c’est la raison pour laquelle il a connu un succès fracassant, toute observation du monde culturel pouvant donner prise à ce type d'explication. Mais les sciences sociales sont avant tout des sciences de l'observation : lorsque les données contredisent les théories, il faut abandonner ces dernières, même à regret.

\title{
La légitimité culturelle en questions $(2 / 2)$
}

\begin{abstract}
"Lisez. Kant attentivement et de plus en plus attentivement et, tout à coup, vous aurez le fou rire, a-t-il dit. D'ailleurs tout original est, à vrai dire, une falsification en soi, a-t-il dit, vous comprenez tout de même ce que je veux dire. Naturellement il y a des phénomènes dans le monde, dans la nature, comme vous voulez, que nous ne pouvons pas ridiculiser, mais en art, tout peut être ridiculisé, tout homme peut être ridiculisé et transformé en caricature, si nous voulons, si vous en avez. besoin, a-t-il dit. Si nous sommes en mesure de ridiculiser, nous ne sommes pas toujours en mesure de le faire, alors le désespoir nous emporte, et ensuite le diable, a-t-il dit. [...] Mais la plupart des gens sont tout de même ridicules, et la plupart des xuvres d'art sont tout de même ridicules, et vous n'avez pas besoin de ridiculiser et de caricaturer" (Thomas Bernhard)
\end{abstract}

"La religion de l'art a aussi ses intégristes et ses modernistes, mais qui s'accordent pour poser la question du salut culturel dans le langage de la grâce" ${ }^{21}$. De fait, tout rapport à l'art ne serait qu'une caricature, une mise en scène illusoire où l'on tenterait, les uns et les autres, de se jouer la comédie tragique de l'amour de l'art. L'amour de l'art n'existe pas, pas vraiment, du moins pas aussi naturellement qu'on pût l'imaginer jusque dans les années soixante-dix, tout imprégnés d'idéologie post-kantienne que nous étions. Depuis lors, sociologues, professionnels de la culture, journalistes ou lecteurs sensibles à divulguer les lieux et les situations où s'exercent subrepticement les pouvoirs symboliques qui perpétuent dans la sublunaire sociale les

${ }^{20}$. Dont William LABOV explorera plus tard divers aspects au travers d'enquêtes socio-linguistiques.

${ }^{21}$ Pierre Bourdieu et Alain Darbel, L'amour de l'art, Les musées d'art européens et leur public, Paris, Les éditions de Minuit, 1969, p. 13. 


\section{La légitimité culturelle en questions -14}

inégalités d'accès à la chose artistique ou culturelle, tous ont été amadoués au moins une fois dans leur vie avec plus ou moins de force par la sociologie de la légitimité culturelle théoriquement raffinée et déclinée dans l'œuvre de Pierre Bourdieu. De "l'amour" aux Règles de l'art, l'ambition de l'auteur vise à proposer un point de vue holistique et donc, englobant, où se rejoignent une sociologie des œuvres et une sociologie des publics rompues, par le biais d'une analyse scientifique, à dévoiler ce qui rend socialement l'cuvre d'art nécessaire, prenant en charge du même coup les fonctions qu'elle remplit et les conditions de maintien des inégalités sociales d'accès qu'elle ménage ${ }^{22}$. L'expérience du beau n'est pas une expérience susceptible d'être universellement partagée par l'humanité sous le seul prétexte qu'il s'agit d'une expérience humaine : en renversant catégoriquement le système kantien, Pierre Bourdieu instruit le procès d'un rapport à l'art et d'une valeur de l'art axiomatisés par les cultures les plus autonomisées dans leurs jugements de goût et dans leurs pratiques, c'est-à-dire les cultures savantes.

Les logiques d'appréciation des acteurs sociaux évoluent là dans une sorte de pré carré clôturé par la conditionnalité des jugements et des pratiques qui porte en creux et systématiquement l'empreinte des classes dominantes. En conséquence, le "sens pratique" des individus les conduit à se comporter selon des schèmes opératoires intégrés - les habitus - dont ils semblent ne pas avoir directement conscience, condition sine qua non de leur opérationnalité sociale et relative garantie de leur efficacité symbolique durable. En partant de ce qui vient tantôt comme un postulat théorique, tantôt comme une conclusion pratique, le sociologue Bourdieu aboutit à l'élaboration d'un panorama d'où émergent les lignes de force qui "magnétisent" et délimitent les marges de liberté dans lesquelles se jouent et se métamorphose la pratique du social ou si l'on veut le social de la pratique des individus. Comme le remarque Bernard Lahire, "Bourdieu a construit en grande partie sa théorie de la pratique [...] contre l'idée d'une pratique orientée rationnellement, intentionnellement, volontairement vers des fins explicites, contre l'idée d'une réflexivité, d'une conscience consciente, systématique et calculatrice.

\footnotetext{
${ }^{22}$ C'est en partant principalement du concept de champ que Pierre Bourdieu explique les réglementations de la nécessité sociale de la culture. On pourra topographier avec profit les apports de l'approche de Bourdieu au regard des autres sociologies de l'art en lisant l'article de Jean-Louis Fabiani "Sur quelques progrès récents de la sociologie des ceuvres" publié dans le n 11 de la revue Genèses.
} 


\section{La légitimité culturelle en questions -15}

Le rapport pratique à la pratique est ainsi défini comme une comprébension immédiate, aveugle à elle-même (une docte ignorance, une conscience non-consciente, sans concept, partielle, floue, non intentionnelle et engagée dans l'urgence de l'action" 23.

De fait, c'est en partie dans l'espace plus ou moins large demeurant entre l'immédiateté de la pratique et le retour réflexif que l'on est capable de formuler sur celle-ci que le sociologue peut échafauder un repérage des ajustements possibles entre les conduites des acteurs sociaux et les représentations qu'ils s'en font. Cette sorte d'économie feuilletée des justifications sociales du sociale mise à jour scientifiquement rendrait sans doute assez justement compte des échanges et des processus d'incorporation culturelle, des intériorisations de l'extériorité et inversement de l'extériorisation de l'intériorité. Le pari interprétatif qui se trame dans la légitimité culturelle exprime - on le pressent - un ordonnancement théorique des rapports socialisés à la culture qu'elle soit entendue au sens des pratiques culturelles comme au sens des phénomènes sociétaux caractéristiques d'une civilisation donnée. Nous n'en discuterons ici ni les bien-fondés, ni même les effets de réalité qu'elle serait susceptible de provoquer. Son ingéniosité reste incontestable pour comprendre les instances systémiques du champ de la culture qui - remarquons-le - se confondent généralement de manière fascinante avec les lieux de la communication culturelle. Notre propos s'intéresse plutôt aux revers de la thèse dont l'harmonie positive tend à éclipser l'énigme sociologique que certaines données empiriques continuent encore de receler sur les territoires de l'enquête.

Ainsi en va-t-il de la liaison parfois problématique et rarement interrogée que la sociologie de la légitimité culturelle établit convoque systématiquement entre les variables de diplôme et les pratiques culturelles qui trouveraient dans ces dites variables une partie de leur moteur social. Apparemment, à en croire Bourdieu, les agents sociaux ne peuvent " faire" leur culture que dans la mesure où ils sont dotés d'habitus ajustés au champ, c'est-à-dire au sens du jeu comme capacité d'anticiper sur le mode pratiques des "à venir que se donnent dans la structure même du jeu, ou autrement dit, dans la mesure où ils ont été ainsi

${ }^{23}$ Bernard Lahire, L'homme pluriel, Paris, Nathan, 1998, p. 171. 


\section{La légitimité culturelle en questions - 16}

constitués qu'ils sont disposés à appréhender dans la structure présente des potentialités objectives qui s'imposent à eux comme des choses à faire "24. Au reste, on comprend que la variable sociologique du diplôme avait presque naturellement toutes les chances de s'instituer comme l'un des révélateurs de l'habitus doté des plus fortes potentialités: certains effets de la pratique permettent pourtant, nous le verrons, d'isoler et de sanctionner quelques paradoxes qu'il produit. Dans le même ordre d'idées, lorsqu'il porte sur une auto-évaluation des acteurs sociaux sur eux-mêmes, le vécu des pratiques repéré dans les enquêtes par questionnaires est souvent sujets à soupçons dans la sociologie de Bourdieu, une sur-évaluation ou une sous-évaluation étant considéré comme une volonté de redorer le blason de ce qu'est dans les faits la pratique réelle.

\section{Temps perçu - temps vécu, une improbable équation}

L'une des illustrations les plus saillantes de cette suspicion est sans doute celle portant sur la comparaison entre le temps "objectif" et le temps "subjectif" d'une visite de musée qui figure dans $L$ 'Amour de l'art. Ce que les auteurs appellent ici le temps objectif, c'est le temps que les visiteurs ont effectivement passé dans un musée ; ce temps est mesuré grâce à un chronométrage de l'heure d'entrée et de sortie réalisé à leur insu par une équipe d'enquêteurs. Le temps subjectif correspond pour sa part au temps que les visiteurs déclarent avoir passé dans le musée. Le tableau qui suit croise la différence entre temps déclaré et temps passé avec les classes sociales populaires, moyennes, hautes.

Du temps déclaré au temps passé

\begin{tabular}{|c|c|c|c|c|c|c|c|c|}
\hline Sans & inférieur & égal & supérieur & de supérieur & deelsupérieur & dede plus de $15^{\prime}$ & total & total \\
\hline
\end{tabular}

\footnotetext{
${ }^{24}$ Pierre Bourdieu, Méditations pascaliennes, Seuil, p. 254.
} 


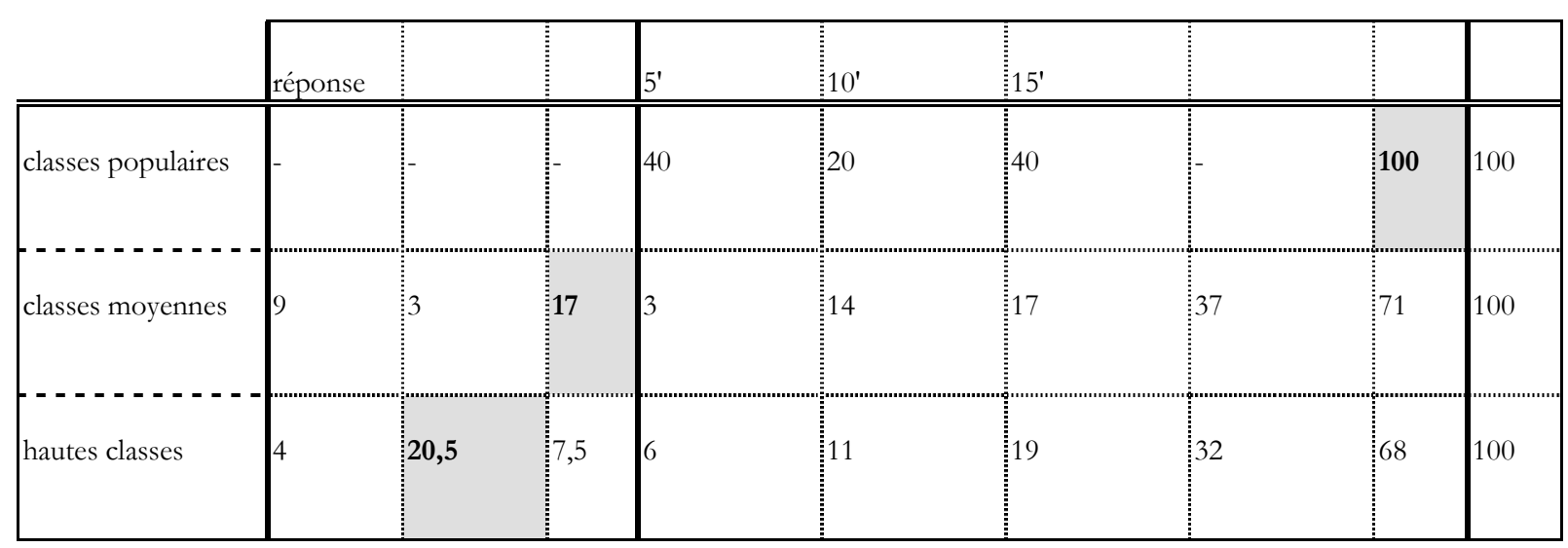

L'amour de l'art ne comporte aucun commentaire explicite consacré à ce tableau. Indexé en tant qu"'enquête annexe de confirmation", il paraît donc raisonnable de le rattacher à la thèse sociologique générale de l'ouvrage qui - on le suppose - contextualise la structuration de l'ensemble des données qui lui sont dévolues. Aussi s'agit-il de s'employer à déceler la manière dont s'incorpore cette pièce de la mesure du temps au sein du puzzle construit par Pierre Bourdieu et Alain Darbel si l'on veut identifier le rôle qu'ils attribuent à cet indicateur et sur la relation que celui entretient avec les populations qu'il concerne. Le raisonnement sociologique a toujours - et surtout dans le cadre d'une analyse secondaire de données - pour fonction de s'interroger "sur les conditions sociales de constitution des populations apparemment les plus naturelles, afin de restituer à l'énonciation un fait qui interfêre en toute généralisation, même et surtout silencieuse, à savoir qu'on n'observe jamais qu'avec une lorgnette, fut-elle la plus sophistiquée et la mieux réglée, que ce qui passe dans son champ" ${ }^{25}$.

Ainsi comme n'importe quel tableau croisé d'analyse statistique, le tableau de Pierre Bourdieu et Alain Darbel résume sous des modalités qualitatives ordonnées hiérarchiquement - la classe, la différence entre temps passé et temps déclaré - les résultats d'une question réglée sur une problématique d'ensemble. En conséquence, il importe de déduire tant de la découpe des modalités ordinales - le temps - que celle des modalités

\footnotetext{
25 Jean-Claude Passeron, "Ce que dit un tableau et ce qu'on en dit", in Le Raisonnement sociologique, op. cit., p. 125.
} 


\section{La légitimité culturelle en questions -18}

nominales - la classe sociale - quel est l'état de la relation que les auteurs ont désiré éprouvé entre les éléments d'une population et les chances qu'ont ces éléments d'adopter un comportement donné. En l'occurrence la mesure de la différence entre le temps de visite passé et le temps de visite perçu tient lieu de vérification et prétend illustrer une différence sociologiquement plus large, celle de l'impression subjective laissée par le musée que l'on suppose elle-même liée à l'appartenance sociale d'origine des individus interrogés.

Compte-tenu de sa neutralité certaine, on pouvait de bon droit penser que Pierre Bourdieu et Alain Darbel tenaient avec l'indicateur temps un splendide révélateur pour prendre à partie et recontextualiser l'idée selon laquelle que "le rendement social de la culture artistique dépend au moins autant de l'aptitude d'exprimer les expériences artistiques que de la qualité intrinsèque et invérifiables de ces expériences" ${ }^{\prime 26}$. Malheureusement et si l'on s'en réfère au titre général de l'enquête "Mesure expérimentales des temps de visite et des connaissances picturales", c'est une idée plus large - les effets de la légitimité culturelle -qui va ici remorquer derrière elle la lecture du tableau et non, comme on aurait pu l'espérer, le tableau maigriot ${ }^{27}$ qui érafle la thèse centrale. Attachés à démonter cette prétention du sens commun d'après laquelle "comme tout amour, l'amour de l'art répugne à connaître ses origines et, aux conditions et aux conditionnements communs, il préfere, à tout prendre, les hasards singuliers qui se laissent toujours interpréter comme prédestination" ${ }^{28}$, Pierre Bourdieu et Alain Darbel alloueront d'emblée au temps une présomption de culpabilité.

Car que signifie exactement cette différence entre temps passé et temps déclaré? Que vaut une échelle de comparaison qui mélange allègrement une objectivité et une subjectivité où par la force des choses, c'est la lecture de la subjectivité qui éclate et triomphe? Qu'est-ce

\footnotetext{
26 Ibid., p. 103.

27 Nous n'apprendrons rien ici du temps de visite effectif des uns et des autres. Nous ne saurons pas quel groupe social passe modalement plus de temps dans un musée.

${ }^{28}$ Pierre Bourdieu et Alain Darbel, op. cit, p. 161
} 
exactement que le temps d'une visite au musée ? Est-ce comme le considèrent les auteurs un temps qui délimite une activité avec les bornes que sont les temps d'entrée et temps de sortie ou bien est-ce l'addition de la série des durées cumulées devant chaque oeuvre? Difficile de conclure.

\section{Temps subjectif et légitimation comportementale}

Les caractères du tableau imprimés en gras et donc soulignés par les auteurs censés orienter notre déchiffrage du propos indiquent que 100\% des classes populaires déclarent passer plus de temps que ce que dure effectivement leur visite. 17\% des classes moyennes évaluent justement leur temps passé au musée et 20,5\% des hautes classes pensent qu'ils y sont restés moins longtemps. À première vue Pierre Bourdieu et Alain Darbel font abonder leur chiffre vers une interprétation toute proche du psychologisme de Fraisse dont nous parlions plus haut. Grossièrement si l'on tend à déclarer plus de temps que ce qu'on a réellement passé c'est parce que l'activité à laquelle on était occupé ne nous passionnait guère et que, par conséquent, le temps nous a semblé plus long; on s'est ennuyé durant la visite, donc ennuyé devant les oeuvres, et si on s'y est ennuyé c'est parce que nous ne détenions pas toutes les clefs requises à leur décodage. Inversement si l'on possède les clefs, on ne s'ennuie pas, et le temps de loisir si difficile à habiter à ceux qui n'en n'ont pas l'habitude nous apparaît plus bref; le temps permettrait ainsi d'objectiver à notre insu des un habitus de classe, une manière d'être trahie non pas par une mauvaise auto-évaluation du temps mais par ce qu'elle révèle plus directement : l'ennui qui foule au pied l'amour de l'art. L'ennui qui prend sur le fait la lutte symbolique que se livrent les classes sociales sur le terrain de la culture et où se consolident leur reproduction.

Le travail du sociologue selon Pierre Bourdieu doit s'entendre comme une analyse des positions relatives occupées les individus dans l'espace social et des relations objectives 


\section{La légitimité culturelle en questions - 20}

entre ces positions : cette démarche qu'il qualifie lui-même de "constructivisme structuraliste" espère, de cette manière, dévoiler d'une part les structures objectives de la société qu'il imagine indépendantes de la conscience et de la volonté des agents, et, d'autre part la genèse sociale des schèmes de perception, et pensée et d'action par lesquels les individus, tour à tour, intérioriseraient (l'babitus) et extérioriseraient ces structures sociales (les champs du social). Appliqué à l'amour de l'art, ce raisonnement nous confronte à une sorte de transposition et de généralisation d'un propos plus ancien : celui de La Théorie de la classe de loisir ${ }^{29}$ de Thorstein Veblen (1857-1929). Dans cet ouvrage, l'auteur s'applique à jeter les bases d'une psychologie économique réaliste depuis une étude renouvelée du comportement humain. Tout en rejetant vigoureusement les thèses de Marx, Veblen en utilise les notions, et notamment la distinction entre infrastructure économique et superstructure culturelle. De leur articulation, il propose une lecture évolutionniste de cette relation où les anciennes valeurs attachées au travail - dignité, productivité, dévouement au groupe social - s'inversent dans un nouvel état social où s'exhibe une déconsidération du travail qui entraîne avec elle l'exaltation de la puissance, la vanité de la richesse et la consommation ostentatoire.

À maintes reprises, Thorstein Veblen soulignera la centralité du gaspillage de l'effort ostensiblement affiché et justiciable de son appartenance à la classe qu'il qualifie de "loisirs". Ne serait-ce pas une survivance de cette ostentation que Pierre Bourdieu nous donne à voir dans l'expression du rapport "naturel" à l'art que compose les classes qu'il désigne comme dominantes ? Et c'est en ce sens que chez Thorstein Veblen comme chez Pierre Bourdieu, l'usage du temps participe pleinement à la preuve de cette légitimation du bon goût qui n'est autre qu'une légitimation de classe. Car gaspiller son temps ou déclarer qu'une visite au musée semble plus courte qu'elle ne l'est réellement renvoie à une signification qui est du même ordre: elle exprime le désintéressement qui gouverne une pratique, et collabore à une stratégie plus générale de distinction exercée à travers cette

${ }^{29}$ Thorstein Veblen, Théorie de la classe de loisir, Paris, Éditions Gallimard, 1970, (éd. Or. : The Theory of the Leisure Class, The Macmillan Company, 1899). 
violence douce qu'est la violence symbolique imposée par les groupes détenteurs du capital culturel le plus lourd et qui, non contents de fréquenter les oeuvres légitimes du patrimoine redoublent leur exhibition en circonscrivant une manière légitime de les fréquenter, exempte de tout ennui.

\section{La co-variation problématique du temps et de la légitimité culturelle}

Néanmoins, on le sait les sciences humaines qui"se résolvent au parti hérö̈que de l'expérimentation franche", ne sont pas toujours en mesure de récolter "comme dans les sciences physiques, la récompense "nomologique" des sacrifices qu'elles consentent sur la richesse du cours du monde" ${ }^{30}$. Et, "le rapport d'une variation sociale à la variable qui permet d'attester rigoureusement ses co-variations mesurables n'est jamais un rapport de pure synonymie" ${ }^{31}$. De fait, si l'on prolonge la logique de l'interprétation du temps de Pierre Bourdieu et Alain Darbel en laissant jouer au temps son rôle de trieur social, on achoppe sur quelques décalages dans sa co-incidence avec la théorie de la légitimité culturelle. Emballés par une confirmation généralisée des effets de la légitimation scolaire et culturelle susceptible de s'exercer avec de forts contrastes sur les lieux de culture, les auteurs en arrivent parfois à reconduire bon nombre de leurs interprétations de variables vers la frontière indigène où cohabitent sans heurts la bonne volonté culturelle des classes moyennes et populaires et leur quête d'un niveau culturel normé par la culture savante, soutenu et flatté par une fréquentation des oeuvres d'art. Cela dit, la lecture du tableau du temps de visite proposée n'est pas la seule vraisemblable et les variations temporelles enregistrées appellent au moins trois remarques :

- Les données retenues dans ce tableau font l'objet - on l'a vu d'un choix partial qui aspire à résumer une attitude depuis la création d'une variable artificielle et soupçonneuse qui

30 Jean-Claude Passeron, op. cit. , p. 132.
31 Ibid., p. 117. 
amalgame une mesure objective et une mesure subjective. Ne pouvions-nous nous contenter de croiser de façon classes sociales et temps passé au musées afin d'obtenir un indicateur propre à neutraliser en partie les effets de commentaires qu'entraîne l'autoévaluation ainsi que le montreront certaines enquêtes ultérieures ${ }^{32}$ ? Que charrie exactement avec elle la variable "classe sociale" ? Pourquoi les visiteurs sont-ils si peu à déclarer leur véritable temps de visite temps qu'ils passent au musée ? En quoi les marges retenues pour différencier les temps déclarés sont-elles significatives?

- D'un point de vue strictement méthodologique, et puisque les visiteurs de musées sont amenés en définitive à émettre via le temps déclaré une sorte de jugement sur leur propre pratique, il peut être intéressant de confronter les sous-entendus interprétatifs distillés dans l'orientation donnés aux résultats avec les 3 principes de questionnement inhérents aux sondages et dénoncés par Pierre Bourdieu dans le célèbre texte de sa conférence de Noroît : "l'opinion publique n'existe pas"33. L'auteur y présente une critique mordante des postulats implicites sur lesquels fonctionnent les enquêtes d'opinion. Indirectement, il y définit un anti-programme méthodologique duquel on peut en négatif déduire un programme adaptable au problème du temps déclaré dans l'amour de l'art.

\begin{tabular}{|c|c|c|}
\hline $\begin{array}{l}\text { anti-programme } \\
\text { sous-tendu par } \\
\text { les sondages d'opinion }\end{array}$ & $\begin{array}{l}\text { anti-programme } \\
\text { adapté au temps } \\
\text { déclaré par les } \\
\text { visiteurs de musée }\end{array}$ & $\begin{array}{l}\text { programme adapté au } \\
\text { temps déclaré par les } \\
\text { visiteurs de musée }\end{array}$ \\
\hline $\begin{array}{l}\text { tout le monde peut avoir } \\
\text { une opinion }\end{array}$ & $\begin{array}{l}\text { tout le monde peut } \\
\text { s'auto-évaluer sur le } \\
\text { temps qu'il passe dans } \\
\text { un musée }\end{array}$ & $\begin{array}{l}\text { l'auto-évaluation sur le } \\
\text { temps n'est pas à la portée } \\
\text { de tous }\end{array}$ \\
\hline
\end{tabular}

\footnotetext{
${ }^{32}$ Voir par exemple le compte rendu d'une enquête au Musée Granet d'Aix-en-Provence dirigée par JeanClaude Passeron et Emmanuel Pedler, Le temps donné aux tableaux, Documents Cercom/Imerec, décembre 1991.

${ }^{33}$ Pierre Bourdieu, Questions de sociologie, Paris, Éditions de Minuit, 1984, pp.222-235.
} 


\begin{tabular}{|c|c|c|}
\hline $\begin{array}{l}\text { toutes les opinions se } \\
\text { valent }\end{array}$ & $\begin{array}{l}\text { toutes les déclarations sur } \\
\text { le temps se valent }\end{array}$ & $\begin{array}{l}\text { les temps déclarés ne } \\
\text { signifient pas tous la } \\
\text { même chose }\end{array}$ \\
\hline $\begin{array}{l}\text { il y a un consensus sur la } \\
\text { question posée }\end{array}$ & $\begin{array}{l}\text { la question sur le temps } \\
\text { de visite prend en } \\
\text { compte la même chose } \\
\text { pour tous }\end{array}$ & $\begin{array}{l}\text { la question sur le temps } \\
\text { passé peut ne pas } \\
\text { s'entendre comme telle } \\
\text { pour tout le monde }\end{array}$ \\
\hline
\end{tabular}

Ce programme idéal - compte tenu des dimensions multiples impliquée dans la question du temps - n'est pas simple à concrétiser et le chemin qu'empruntent Pierre Bourdieu et Alain Darbel pour aller de l'indicateur au concept est plus proche des zones sombres du tableau ci-dessus que des objectifs définis dans la colonne de droite. Et, pour que se réalisent parfaitement les énoncés embrassés par la légitimité culturelle, il est nécessaire que le temps puisse s'adosser sur le second principe "les temps déclarés ne signifient pas tous la même chose". Or, ce dernier peut, dès l'origine du processus d'enquête, prendre une signification différente c'est-à-dire l'instant même où le visiteur interrogé répond car sa bonne foi est susceptible d'entrer en jeu. Deux possibilités : soit il est de bonne foi. Son auto-évaluation traduit honnêtement le sentiment du temps passé au musée et le décalage entre le temps passé et temps déclaré est probant pour confirmer la thèse de l'ennui ou du non-ennui sincères. Soit l'enquêté est de mauvaise foi. Il attache une importance au déclaratif en tentant de maîtriser devant l'enquêteur le temps de sa pratique muséale et se surévalue pour la réifier en pratique de distinction puisque de toute façon il ne sait pas qu'il a été chronométré à son insu... Là encore, il est difficile de trancher d'autant que l'interprétation qui fermente dans l'idée de la légitimité culturelle spécule simultanément sur les deux registres. De plus trier sur ce déclaratif le bon grain de l'ivraie n'est pas simple car il ne faut pas négliger le fait que notre échantillon peut être composé d'individus ayant des inclinations pour l'une ou l'autre tendance. Les indicateurs statistiques sont ainsi faits. Si la théorie qu'ils sont censés éclairer est trop généralisante, ils pourront toujours être retournés 


\section{La légitimité culturelle en questions -24}

à son profit. La théorie de la légitimité culturelle est de cet ordre. Elle est boulimique et se nourrit de tout, se transpose à importe quel objet et l'intelligibilité qu'elle livre à notre compréhension donne parfois, si l'on y prend garde, de se suffire à elle-même : en dramatisant le complot culturel où les classes sociales réclament symboliquement leur reconnaissance symbolique, elle travaille sempiternellement sur un ressort ambigu qui administre cette sensation intrigante que jamais ne pourra se résorber l'impasse qui sépare énigmatiquement l'homme des cultures savantes et l'homme des cultures populaires.

- Si l'on se reporte au tableau initial, sans miser, cette fois, sur la légitimité, que l'on se contente d'un dénombrement, le constat s'écarte quelque peu de celui de Pierre Bourdieu et Alain Darbel ; il montre que, tendanciellement, la plupart des visiteurs sur-évaluent leur temps de visite et entre autre $71 \%$ des classes moyennes et $68 \%$ des classes supérieures. De surcroît, il n'y a que dans ces classes sociales que l'on rencontre des écarts supérieurs d'au moins quinze minutes! Faut-il en conclure que ce sont ces fractions de population qui s'ennuient le plus au musée ou qui font preuve d'une plus grande mauvaise foi en opérant cette majoration tactique enregistrée à leur insu à la douane sociologique ? Ou bien faut-il prendre comme telle cette lecture plurielle des temps en admettant que les découpes ne sont pas aussi nettes qu'on les imaginait, qu'elles ne vont pas sans ces contradictions qui demeureront invisibles tant que ne sont pas remises en question la variable omniexplicative du niveau de diplôme derrière laquelle toutes les autres - variable de classe en tête - semblent emboitter le pas. Si d'aventure on redistribue les effectifs de base du tableau de Pierre Bourdieu et Alain Darbel pour ne s'intéresse qu'à la répartition statistique comparée de ceux qui se sur-évaluent, on est en mesure de formuler des énoncés modifiés.

\begin{tabular}{|ll|l|l|l|l|}
\cline { 2 - 5 } \multicolumn{1}{c|}{} & supérieur de 5' & Supérieur de $10^{\prime}$ & supérieur de $15^{\prime}$ & Ide plus de 15' & total \\
\hline \hline classes & 40 & 20 & 40 & & 100 \\
\hline
\end{tabular}




\begin{tabular}{|c|c|c|c|c|c|}
\hline populaires & & & & & \\
\hline classes & 3 & 19 & 26 & 52 & 100 \\
\hline $\begin{array}{l}\text { hautes } \\
\text { classes }\end{array}$ & 8 & 16 & 29 & 47 & 100 \\
\hline
\end{tabular}

Ce sont les classes populaires qui s'auto-évaluent le mieux. Elles n'excèdent jamais les 15 minutes de différence. Il ne se dégage aucune régularité dans leur manière de la faire. Les classes moyennes sont celles qui se surévaluent le plus. Cette sur-évaluation est aussi régulière dans sa progression que celle que l'on enregistre pour les classes supérieures où près de 50\% des individus majorent leur temps de visite de plus de 15 minutes.

Que déduire de ces remarques si on les rapproche avec celles des auteurs de l'Amour de l'art? Avant toute chose que si l'indicateur temps contextualise très sensiblement des comportements, il ouvre sur une polysémie interprétative d'où filtre une complexité du social qui devrait logiquement nous obliger à nous rapprocher des pratiques effectives et de ce qui constitue l'objet de ces pratiques. Etablir par exemple que le classes supérieures concourent à la fois vers une sur-évaluation de et une sous-évaluation de leur temps de visite fournit en-soi un énoncé sémiologique et sociologique où culture et usage du temps des publics méritent un approfondissement particulier. Car, quoi qu'il en soit, il ne faut pas négliger ce fait tautologique que semblent ignorer les auteurs de L'Amour de l'art : les publics du musée sont d'abord et avant tout des publics ! C'est-à-dire qu'ils sont toujours réunis par un intérêt commun qu'on ne peut leur dénier, même si cet intérêt est diversement vécu : les filtres sociaux économiques et culturels qui ont conduit ces publics toutes classes confondues - à la porte du musée sont extrêmement nombreux et ne doivent pas être trop écrasés. Et, comme l'écrit Jean-Claude Passeron : "la sociologie de la culture a commencé $[. .$.$] à faire un sort à ce qui se donne à voir comme "important", comme "significatif" dans le$ champ de l'bistoire des formes, des mécanismes de leur démarcation ou dans leur manifestations de leur 


\section{La légitimité culturelle en questions - 26}

pouvoir social. C'est là dessus qu'elle a réglé sa doctrine des structures et des fonctions symboliques. Les choix conceptuels qui commandent la description des conduites symboliques ainsi que les choix méthodologiques qui déterminent la technique d'observation (sélection des terrains de pratiques, catégorisation des différences, repérages des cooccurrence) portent toujours la marque des "objets", c'est-à-dire des "traits pertinents", auxquels s'est insidieusement accoutumée une sociologie de la culture centrée - par dilection ou animadversion peu importe - sur les pratiques les plus valorisées des groupes dominants ou des groupes intermédiaires qui réferent exclusivement leurs symbolismes à la reconnaissance sinon à la connaissance sinon à la connaissance de la légitimité culturelle" ${ }^{\prime 34}$. En ébranlant quelque peu les catégorisations rituelles de la sociologie des oeuvres, les temporalités multiples - du spectateur à l'oeuvre qu'il contemple - parce qu'elles traversent les modes de constitution des pratiques, ouvrent aussi - dès l'instant où le temps y est symboliquement appréhendé une des voies possibles pour repenser avec la prudence les conclusions courantes des études de consommation culturelle.

\footnotetext{
${ }^{34}$ Jean-Claude Passeron, Le Savant et le populaire, Éditions des Hautes Études-Gallimard-Le Seuil, 1989, p. 22 .
} 